\title{
A Survey on Scheduling Algorithms in Cloud Computing
}

\author{
Mrs.M.Padmavathi ${ }^{1}$, Shaik. Mahabbob Basha, Professor ${ }^{2,}$ \\ Mr.Srinivas Pothapragada, CEO of Ostilio ${ }^{3}$ \\ ${ }^{I}$ Department of computer science and engineering Swarna Bharathi Institute of Science and Technology, \\ Pakabanda Bazaar, Khammam, Telangana. \\ ${ }^{2}$ Al-Habbeb College Of Engineering And Technology Chevella,R.R District,501503,Hyderabad,Telanagana \\ ${ }^{3}$ Mr. Srinivas Pothapragada, CEO of Ostilio, RegAdd: Kanajiguda, Secunderabad, Telangana, 500015
}

\begin{abstract}
Cloud Computing is every Where .Cloud Computing gets its name as metaphor for the Internet and it has changed the model of storing and managing data for scalable, real time, internet based applications and Resources satisfying end users needs. Cloud computing services are usually backed by large-scale data centers are built to serve many users and many disparate Applications, for this we are considering Virtualization a s a perfect match. Resource Scheduling is a complicated task in cloud computing environment because there are many alternative computers with varying capacities. in this paper a review was performed on the Existing Scheduling Algorithms. limitations with existing Systems was list out. As a future enhancement a new Algorithm will develop.
\end{abstract}

Key words: Cloud Computing, Virtualization, CPU Scheduling algorithms

\section{Introduction:}

Cloud computing is an approach to computing that builds on virtualization's efficient pooling of resources to create an on-demand, elastic, self-managing virtual infrastructure that can be allocated dynamically as a service. Virtualization enables you to create a dynamic and flexible datacenter, and can reduce operating expenses through automation while also reducing planned and unplanned downtime. Virtualization, in addition to being the underlying technology for cloud computing, enables organizations of all sizes to make improvements in the areas of flexibility and cost containment. Virtualization is a process that breaks the hard connection between the physical hardware and the operating system and applications running on it.

Applications in cloud environment are deployed in Remote Data Centers (DC's) where servers with high capacity and Storage systems are located.

Using cloud services, cloud users can deploy a wide variety of applications dynamically and on demand usually sddressed from three fundamental aspects:Infrastucture as a Service(Iaas),Platform as aService(Paas) and Software as a Service(Saas)[4].Most cloud Service Provides use Machine Virtualization to provide flexible and cost effective Resource Sharing.it is the responsibility of the cloud service providers to manage its Resources in an efficient way to make the needed resources available on demand to the cloud users[4].

\section{Literature Review:}

GenerallyResource allocation and scheduling of resources have been an important aspect that affects the performance of networking, parallel, distributed computing and cloud computing. Scheduling process in cloud can be generalized into three stages namely[2]

Resource discovering and filtering:Datacenter Broker discovers the resources present in the network system and collects status information related to them.

Resource selection:Target resource is selected based on certain parameters of task and resource. This is deciding stage.

Task submission:Task is submitted to resource selected.

Resource Scheduling is a complicated task in cloud computing environment because there are many alternative computers with varying capacities. Resource allocation task is scheduled for the Process which gives the available resources and user preferences. In order to efficiently allocate computing resources; Efficient task scheduling mechanism can meet users' requirements and improve the resource utilization. The following process has to follow in Resource Scheduling(fig 1): 
Fig 1: Resource Scheduling in Cloud Computing Environment

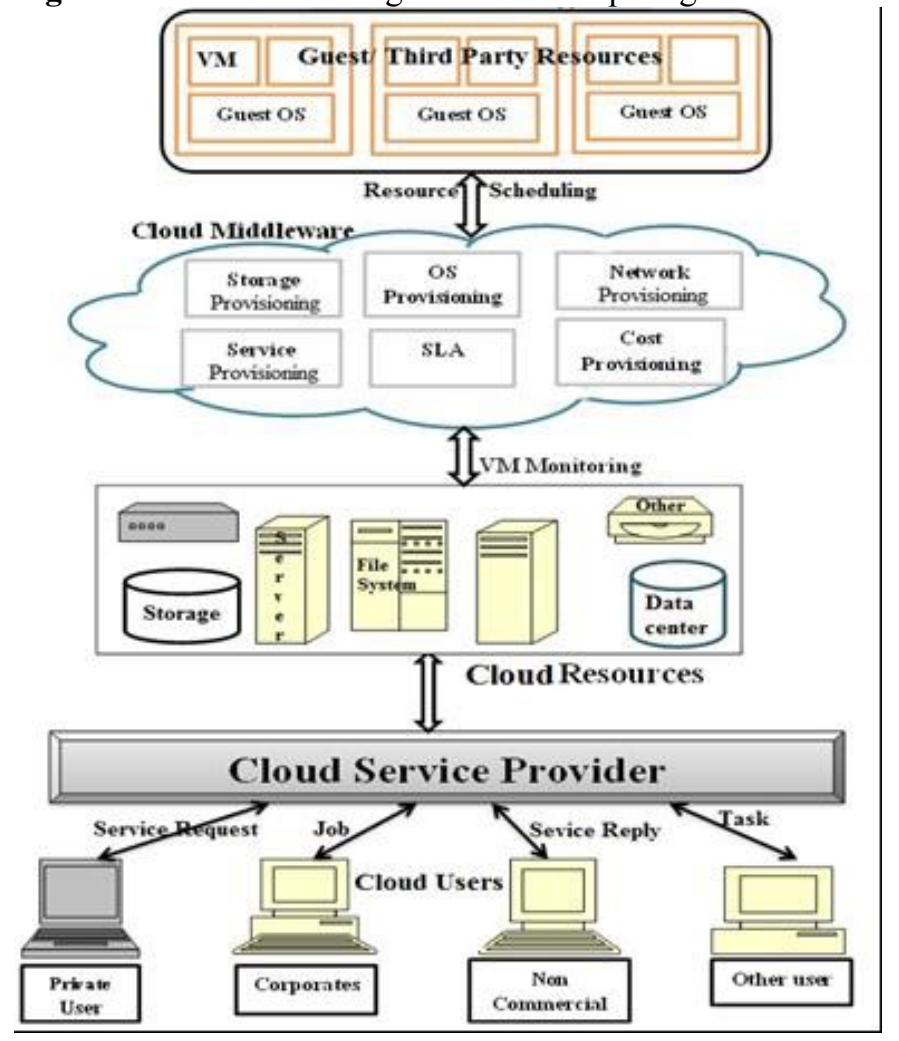

The following are the seven Major factors in Scheduling:

1.Throughput Time

2.Turn Around Time

3. Waiting Time

4.Response Time

5.Utilization of CPU Time

6. Computational Complexity (job length, processing power)

7.Computing Cost(processor cost)

The Scheduler maintains the balances between QOS and fairness among the jobs so that the efficiency may be increased. Since the number of cloud users growing exponentially and the demand resources like virtual machines also increased. So the scheduling is a most critical issue in cloud computing The main target of scheduling is to maximize the resource utilization and minimize processing time of the tasks[3].

An efficient job scheduling strategy must aim to yield less response time. so that the execution of submitted jobs takes place within a stipulated time and simultaneously there will be an occurrence of in-time resource reallocation. The primary goal of Cloud Computing is to provide efficient access to remote and geographically distributed resources with the help of Virtualization in Infrastructure as a Service (IaaS). We need various kind of virtual machines (VM) as per the requirement and cloud provider provides these services as per the Service Level Agreement (SLA) to ensure QoS[3].

\subsection{Scheduling Techniques:}

2.1.1 .Preemptive /Non-Preemptive Techniques

In Preemptive Scheduling Algorithm, the job which is in processing can be Stopped or Postponed /in Non-preemptive the job which is in execution can not be stopped until the program execution completes.

\subsubsection{Static/Dynamic Priority Algorithms}

in case of Static Priority Algorithms Priority can not be changed, where as in case of Dynamic Priority Algorithm we can modify the Priority.

According to a simple classification[1], job scheduling algorithms in cloud computing can be categorized into two main groups are Batch mode heuristic scheduling algorithms (BMHA) and online mode heuristic algorithms(OMHA). In BMHA, Jobs are queued and collected into a set when they arrive in the 
system. The scheduling algorithm will start after a fixed period of time. The main examples of BMHA based algorithms are; First Come First Served scheduling algorithm (FCFS), Round Robin scheduling algorithm (RR).

By On-line mode heuristic scheduling algorithm, Jobs are scheduled when they arrive in the system. Since the cloud environment is a heterogeneous system and the speed of each processor varies quickly, the online mode heuristic scheduling algorithms are more appropriate for a cloud environment[1] .

There are number of CPU scheduling Algorithms available, but it is difficult to decide which one is the best Algorithm .let us have a review on job scheduling Algorithms in Cloud Computing.

\subsection{Round robin}

\section{Analysis of Existing Algorithms}

It is the simplest algorithm that uses the concept of time quantum or slices. Here the time is divided into multiple slices and each node is given a particular time quantum or time interval and in this quantum, the node will perform its operations. The resources of the service provider are provided to the client on the basis of this time quantum. In Round Robin Scheduling the time quantum play a very important role for scheduling, because if time quantum is very large then Round Robin Scheduling Algorithm is same as the FCFS Scheduling. If the time quantum is extremely too small then Round Robin Scheduling is called as Processor Sharing Algorithm and number of context switches are very high[1].

\subsection{Shortest Response Time First}

The basic idea is straightforward: each process is assigned a priority, and priority is allowed to run. Equal-Priority processes are scheduled in FCFS order. The shortest-Job-First (SJF) algorithm is a special case of general priority scheduling algorithm. An SJF algorithm is simply a priority algorithm where the priority is the inverse of the next CPU burst. That is, the longer the CPU burst, the lower the priority and vice versa. Priority can be defined either internally or externally. Internally defined priorities use some measurable quantities or qualities to compute priority of a process[1].

\subsection{The minimum Completion time job Scheduling Algorithm:}

it attempts to allocate the selected job to the available VM that can offer the minimum Completion time Taking in to account its current Load.the main Criterion to determine the VM in the minimum completion time Scheduling Algorithm is the processor speed and the current Load on each VM.The Algorithm First Scans the available VM s in order to determine the most appropriate machine to perform the job.Subsequently it dispatches the job to the most Suitable VM and starts Execution[3].

\subsection{LCFPE and SCPF Al;gorithms}

The Algorithms are designed for private cloud environment where the Resources are limited. The first algorithm is named Longest Cloudlet Fastest Processing Element(LCFPE) which considers the computational complexity of the Cloudlets in the process of making scheduling decisions.SCPF The second algorithm is named Shortest Cloudlet Fastest ProcessingElement(SCPF)[5].

\subsection{1 .LCPF[5]}

- Sort the Cloudlets in descending order of length

- Sort the Processors in descending order of processing power

- Map the Cloudlets from sorted list to the sorted list of processors on one-to-one mapping basis

\subsubsection{SCPF[5]}

- Sort the Cloud lets in Ascending order of length

- Sort the processors in descending order of processing power.

- Map the jobs from sorted list to the sorted list of processors on one-to-one mapping basis

\subsection{Genetic Algorithm (GA)}

GA is a search heuristic that mimics the process of natural selection. This heuristic (also sometimes called a meta heuristic) is routinely used to generate useful solutions to optimization and search problems. Genetic algorithms belong to the larger class of evolutionary algorithms (EA), which generate solutions to optimization problems using techniques inspired by natural evolution, such as inheritance, mutation, selection, and crossover.

Genetic algorithms find application in bioinformatics, phylogenetics, computational science, engineering, economics, chemistry, manufacturing, mathematics, physics, pharmacometrics and other fields. Genetic Algorithm Applied in private cloud. 
3.5.1 Standard Genetic Algorithm(SGA)[5]

- Produce an initial population by randomly generated individuals

- Evaluate the fitness of all individuals

- while termination not to met do

- select fitter individuals for reproduction

- crossover between individuals

- mutate individuals

- evaluate the fitness of the modified individuals

- Generate a new Application

- End While

3.5.2 Modified Genetic Algorithm (MGA)[5]

It is developed by modifying the initial population with LCFP,SCFP and by controlling the stochastic operators of standard genetic algorithm[5].

- Generate an initial population of individuals with output of schedules of Algorithms LongestCloudletto Fastest Processor(LCFP),Smallest Cloudlet To Fastest Processor (SCFP) ans 8 Random Schedules.

- Evaluate the fitness of all Individuals

- While termination Condition not met do

- select fitter individuals for Reproduction with minimum Execution time

- cross over by two individuals by two point crossover

- mutate individuals by simple swap operator

- Generate a new Application

- End While

*Here cloud let Refers to user jobs in Cloud Computing.

\subsection{Ant Colony Algorithms}

Ant Colony Algorithms family, in swarm intelligence methods, and it constitutes some meta heuristic optimizations. Initially proposed by Marco Dorigo in $1992 \mathrm{in} \mathrm{his} \mathrm{PhD}$ thesis and it is based on the behavior of real ants in 1996[6][7], it is a new heuristic algorithm for the solution of combinatorial optimization problems. Investigations show that: Ant has the ability of finding an optimal path from nest to food. On the way of ants moving, they lay some pheromone on the ground; while an isolated ant encounter a previously laid trail, this ant can detect it and decide with high probability to follow it. Hence, the trail is reinforced with its own pheromone. The probability of ant chooses a way is proportion to the concentration of a way's pheromone. To a way, the more ants choose, the way has denser pheromone, and the denser pheromone attracts more ants. Through this positive feedback mechanism, ant can find an optimal way finally [6][7].

\subsubsection{ACO(Ant Colony OPtimization)}

It is Random optimization Search approach that will be used for allocating the incoming jobs to the Virtual machines.

ACO is inspired from the ant colonies that work together in foraging behavior. In fact the real ants have inspired many researchers for their work, and the ants approach has been used by many researchers for problem solving in various areas. This approach is called on the name of its inspiration ACO. The ants work together in search of new sources of food and simultaneously use the existing food sources to shift the food back to the nest. The ant use two types of pheromone

for its movement these are [8]

\subsubsection{Foraging Pheromone (FP)}

In a typical ACO the uses foraging pheromones to explore new food sources. In our algorithm the ant would lay down foraging pheromone after encountering under loaded nodes for searching overloaded nodes. Therefore, after an ant comes up to an under loaded node it will try to find the next path through foraging pheromone [8].

\subsubsection{Trailing Pheromone (TP)}

In a typical ACO the ant uses trailing pheromone to discover its path back to the nest. However, in our algorithm the ants would use this to find its path to the under loaded node after encountering overloaded node. Therefore, after an ant encounters an overloaded node it will try to trace back[ 8].

The main aim of the two types of pheromone updation is to classify the ants according to the types of nodes they are currently searching for. The ants after originating from the head node, by default follow the 
Foraging pheromone, and in the process, they update the FP trails. After coming upon an overloaded node they follow the Trailing Pheromones and simultaneously update the TP trails of the path. After reaching an under loaded node of the same type they update the data structure so as to move a particular amount of data from the overloaded node to under loaded node. Ants then select a random neighbor of this node, and if they encounter an under loaded node they start following the FP to trace an overloaded node, therefore they repeat the same set of tasks repeatedly in a network to improve the network performance[8].

Ant Colony algorithm is a random search algorithm ,in TSP(TravellingSalesPerson) problem study well applied, TSP problem is a given $\mathrm{n}$ cities and a sales man from a city to visit the city through one and only one last return to the starting poing of the Shortest path.

3.6.2 MACOLB(ModifiedAntColonyOptimizationforLoadBalancing) Decreases the degree of imbalancing between available virtual machines and increases the overall performance.[9]

- Module-I Find Overloaded node.

- Module-II Select Virtual machine of overloaded node

- Module-III Placed the virtual machine with other node.

MACOLB modified the concept of Ant colony optimization in term of movement of the ant that is in both forward direction and backward direction. The way in which ants are created pheromone table that contains the information about all nodes and its corresponding load. In this algorithm balance acquired between the node with efficiency \& maximum utilization of resource in this ACO algorithm.

It also improves the performance by achieving the good result in terms of throughout, response time, less energy consumption. Therefore, this algorithm for load balancing in cloud Computing plays a very important role in future. This algorithm can be applied to balance the load in clouds also the performance can also be increased by varying different parameters which would be scope for future research[9]

\section{Limitations With Existing Systems}

There is an additional load on the scheduler to decide the size of quantum and it has longer average waiting time, higher context switches higher turnaround time and low throughput. The round robin scheduling in the cloud is very similar to the round robin scheduling used in the process scheduling. the major drawback of using this algorithm is that the power consumption will be high as many nodes will be kept turned-on for a long time

One major difficulty with SJF is the need to know or estimate the processing time of each job (can only predict the future!)Also, long running jobs may starve; because the CPU has a steady supply of short jobs. with the minimum Completion time job Scheduling Algorithm also user has to estimate the length of the job.they are not considering the perfect utilization of Resources ,Economic factor .

\section{Conclusion And Future Enhancement}

As I Performed thorough study of the above Scheduling Algorithms and can conclude that according to the requirement the user has to select the Scheduling Algorithm., but they are with static in nature , lack in Reliability, no Elasticity and they are not considering load balance.. In My Future Enhancement will propose a new Job Scheduling algorithm which is Dynamic, Elastic, Reliable, Scalable and load balancing. i will perform the comparative study between the proposed Scheduling Algorithm and Existing Scheduling Algorithms. The proposed Algorithm Performance will be evaluated by using Cloud Sim Tool Kit.

\section{Acknowledgements}

The author would like to acknowledge and express their heartfelt gratitude to the Swarna Bharathi Institute of Science and Technology [SBIT] Management, Chairman, Director, Principal and Dean R\&D. for their vital encouragement, support and facilities providing by them achieve this task and at they would like to thank Anonymous Reviewers for their valuable suggestions and comments. This paper has greatly benefited from their Efforts.

\section{References}

[1]. Vignesh V, Sendhil Kumar KS, Jaisankar N "Resource Management and Scheduling in Cloud Environment”International Journal of Scientific and Research Publications, Volume 3, Issue 6, June 20131 ISSN 2250-3153,PP:1-4

[2]. Dr. Amit Agarwal, Saloni Jain "Efficient Optimal Algorithm of Task Scheduling in Cloud Computing Environment" International Journal of Computer Trends and Technology (IJCTT) - volume 9 number 7- Mar 2014,PP:345-349

[3]. IM.Vijayalakshmi, IIV.Venkatesa Kumar "Investigations on Job Scheduling Algorithms in Cloud Computing" International Journal of Advanced Research in Computer Science \& Technology (IJARCST 2014) Vol. 2 Issue Special 1 Jan-March 2014 , ISSN : 2347 8446 (Online) ,ISSN : 2347 - 9817 (Print),PP:157-161 
[4]. Arabi E.Keshk,Ashraf El-SIsi,Medhat A.Tawfeek,F.A.Torkey "Intelligent Strategy of Task Scheduling in Cloud Computing for Load Balancing” International Journal of Emerging trends \& Technology in ComputerScience(ITETTCS)Vol 2,Issue 6,NovemberDecember 2013,ISSN 2278-6856,PP:12-22

[5]. Shaminder Kaur, Amandeep Verma "An Efficient Approach to Genetic Algorithm for Task Scheduling in Cloud Computing Environment” I.J.Informational Technology and Computer Science,2012,10.PP 74-79.

[6]. M. Dorigo, M. Birattari and T. Stutzle," Ant Colony Optimization-Artificial Ants as a Computational Intelligence Technique" , IEEE Computational Intelligence Magazine,1556-603X,2006, pp. 28-39.

[7]. Jaskiran kaur Inderpal Singh," A Survey on Ant colony Optimization ”,International Journal of Computer Science \& Engineering Technology (IJCSET) ISSN : 2229-3345, IJCSET Vol. 4 No. 06Jun 2013 ,pp:713-718.

[8]. R. Rastogi Kumar Nishant, Pratik Sharma,, "Load Balancing of Nodes in Cloud Using Ant Colony Optimization." Proceedings of the 14th International Conference on Computer Modelling and Simulation (UKSim), March 2012, IEEE, pp: 3 -8,

[9]. Shagufta Khan, Niresh Sharma,"Effective Scheduling Algorithm for Load balancing (SALB) using Ant Colony Optimization in Cloud Computing" International Journal of Advanced Research in Computer Science and Software Engineering”, Volume 4, Issue 2,February 2014 ISSN: 2277 128X,PP:966-973
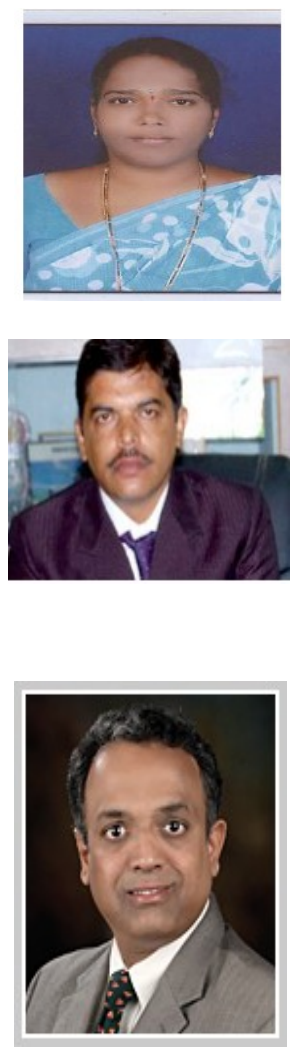

Mrs M.padmavathi working as Asst.Professor in Computer Science and Technology Dept..She Registered her Ph.D from JNTU(H) in the Academic Year 2012-13.Her Research Area is Cloud Computing in that Specialization is ResourceAllocation in Iaas.Up to now Published 8 Articles in international Journals, 2 in National Journals and one in IEEE-Explore

Mahaboob Basha received Engineering Degree In Electronics from Bangalore Univerisy, M.Tech( DS\&CE) from JNTU and Ph.D in Image Processing From S.K.University . Currently He is Professor in Al Habeeb College of Engineering \& Technology, Hyderabad. His research areas include Cloud Computing, Network Security, Image and Video Processing

Mr.Srinivas Pothapragada, CEO of Ostilio is an industry veteran and serial entrepreneur. He was CEO of TidalData. He has over 25 years of industry experience. He is on the board of many US companies, an advisor to numerous International Organizations and is an Investor. $\mathrm{He}$ is the CEO of TidalData, an Application Centric Unified Storage Company. Before TidalData, he co-founded SANOVI in 2002 and headed the company as its President \& CEO. He grew SANOVI to be the market leader in Recovery Management. Sanovi is headquartered out of Boston and its investors include Bayfleet and Advanced Technology Ventures. Srinivas held various senior management positions and led an enterprise product line of Auspex's NetServer series. Before joining Auspex, Srinivas worked at Sun Micro Systems and Sequent (IBM). Srinivas has numerous International Patents to his credit 